\title{
A Multiwavelength Study of the Barred Spiral Galaxy NGC 7479
}

\author{
S. Laine and S. T. Gottesman \\ University of Florida, Astronomy Department, P.O. Box 112055 \\ Gainesville, FL 32611-2055, U.S.A.
}

\begin{abstract}
The detailed investigation of the activity and dynamics in NGC 7479 confirms the importance of understanding individual galaxies. We have discovered an S-shaped emission structure in $21 \mathrm{~cm}$ continuum. This detection together with strong nuclear $10 \mu \mathrm{m}$ and $\mathrm{H} \alpha$ emission may imply the existence of a central massive black hole. We also address the origin of the asymmetric spiral structure in NGC 7479. The lack of any visible companion and the unperturbed $H I$ velocity field suggest that the asymmetry has an internal origin.
\end{abstract}

\section{Introduction}

The study of seemingly isolated barred galaxies should tell us about the effects of the bar on the dynamics of the disk. One of the most interesting objects for this kind of investigation is NGC 7479. It has a very luminous spiral arm to the west of the nucleus. On the eastern side only diffuse spiral structure can be seen. This galaxy has been classified as a starburst, and also as a LINER. Therefore we need to disentangle the effects of a mildly active nucleus and star formation, and understand their origin.

\section{Observations}

1. Optical and near-IR observations. We obtained UKIR'T $J H K$ images of NGC 7479 on 14 July 1994 . The total exposure times were 5 minutes in each of $J$ and $H$ and 10 minutes in $K$. The total field of view is $73.2 \mathrm{x}$ 73.2 arcsec and the pixel size is 0.286 arcsec. Seeing was about 0.8 arcsec. $U B V R I$ band images of NGC 7479 were taken at the $2.5 \mathrm{~m}$ Isaac Newton Telescope on La Palma on 17 August 1990. The seeing was about 2.0 arcsec and the pixel size 0.54 arcsec, covering a total field of $5.2 \times 3.5$ arcmin. NGC 7479 was also observed by Pierre Martin and Daniel Friedli at the Steward Observatory $2.3 \mathrm{~m}$ telescope on Kitt Peak 13 October 1993 in $B, V, R$, and $I$ bands. The seeing was around 1.1 arcsec and the pixel size 0.3 arcsec, covering a field of $1.9 \times 2.9$ arcmin. $\mathrm{H} \alpha$ images and spectra were obtained by Stuart Vogel using the Maryland-Caltech FabryPerot spectrometer at the $1.5 \mathrm{~m}$ telescope of the Palomar Observatory in September 1994. Deep exposures with varying resolution (3.6 arcsec - 11.8 arcsec) were made. 


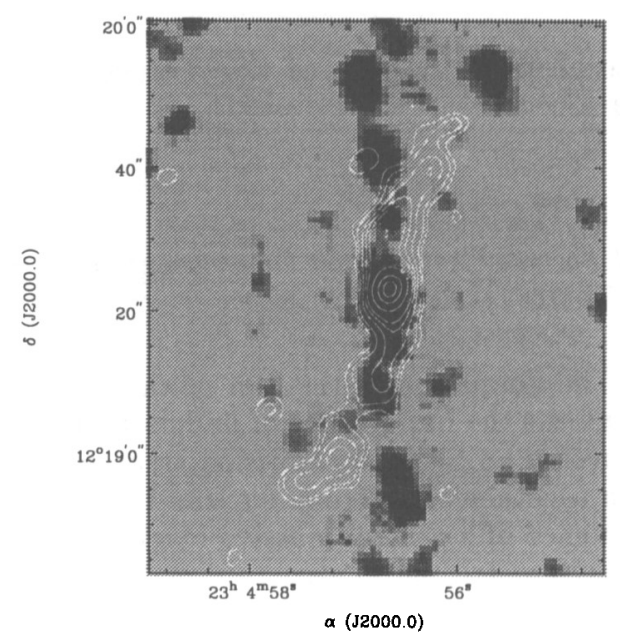

Figure 1. The $21 \mathrm{~cm}$ radio continuum emission contours on an $\mathrm{H} \alpha$ grayscale image

The western arm has a very blue color. The change of color from red to blue at the southern end of the bar is very abrupt. A dust lane follows the center of the bar, slightly displaced toward the leading edges of the bar. $\mathrm{CO}$ and $\mathrm{H} \alpha$ emission occur along this ridge, which we interpret as a shock. $\mathrm{H} \alpha$ emission is concentrated in the spiral arm branches northeast of the bar, the bar itself and the western spiral arm.

2. CO observations. NGC 7479 was observed at Owens Valley $\mathrm{mm}$ interferometer in three different configurations between October 1994 and January 1995. The achieved spatial resolution is 2.5 arcsec and the velocity resolution is $12.7 \mathrm{~km} \mathrm{~s}^{-1}$. One sigma noise value is $0.1 \mathrm{mJy} \mathrm{beam}^{-1}(=0.16$ $\mathrm{K})$. Emission was detected only in the bar and small areas near the end of the bar. The CO emission spans a width of $400 \mathrm{~km} \mathrm{~s}^{-1}\left(570 \mathrm{~km} \mathrm{~s}^{-1}\right.$ when corrected for 45 degree inclination). In the central 10 arcsec the elongated structure is interpreted as a nuclear bar. The CO velocity field has a peculiar inverse S-shape in the nucleus, which could be a combination of streaming along the bar and circular rotation in a nuclear disk.

3. H I observations. C configuration observations of NGC 7479 were made by J. Kenney in September 1989 at the VLA. We observed NGC 7479 in the B configuration in August 1994 and in the D configuration in June 1995. The resulting resolution is about 6 arcsec. The atomic hydrogen disk looks relatively undisturbed in the VLA C configuration observations. The most surprising feature detected in the $\mathrm{B}$ configuration observations was an S-shaped radio continuum feature around the nucleus (Figure 1). It is not spatially coincident with any optical, near-infrared or $\mathrm{mm}$ emission. Atomic hydrogen is concentrated in the western arm and northeast of the 
northern end of the bar. The H I arms extend well beyond the optical arms.

\section{Numerical simulations}

A tree-SPH code kindly provided by Drs. Heller and Shlosman has been used to simulate the gas behavior in the barred potential. The analytical fit to the potential of the galaxy has been provided by Dr. Alice Quillen, and is based on a $K$ band image of NGC 7479 . We start with 12288 "gas particles" and evolve the models for 24 dynamical times. During this time the nonaxisymmetric part of the potential is gradually turned on. We vary the pattern speed of the bar, and take the gas self-gravity and star formation into account. The tentative results indicate that the corotation must lie beyond the end of the bar to achieve a good fit with the $\mathrm{CO}$ observations.

\section{Discussion}

At least two scenarios could explain the observations of NGC 7479. A fairly recent interaction may have caused the asymmetric spiral structure, the high abundance of molecular gas in the nucleus and the long arms seen in the H I gas. However, no companion is seen. Possibly the perturber has merged with NGC 7479. In this case the perturber has been tidally disrupted. But the unperturbed H I velocity field suggests that the observed features are a result of intrinsic dynamical evolution. The gas flows into the nucleus until a critical density is reached and a starburst takes place. Earlier studies of the stellar colors suggest that the latest starburst in NGC 7479 took place approximately $10^{9}$ years ago. The great abundance of circumnuclear molecular gas may indicate that NGC 7479 is on the verge of the next burst.

One of the most puzzling features is the radio continuum emission. The "bent jet"-like morphology may imply a nuclear supermassive black hole, supported by the LINER classification of the nucleus. The UV radiation from the central object heats the circumnuclear dust and gas, causing the high $10 \mu \mathrm{m}$ luminosity and the nuclear $\mathrm{H} \alpha$ emission.

Acknowledgments. We express our gratitude to several colleagues, most notably Elias Brinks, Daniel Friedli, Clayton Heller, Jeff Kenney, Johan Knapen, Mercedes Prieto, Alice Quillen, Mike Regan, Isaac Shlosman, Antonia Varela, Stuart Vogel and Min Su Yun for sharing their data with us and their invaluable help with the observations, data reduction, modeling and analysis. The VLA of the National Radio Astronomy Observatory is operated by Associated Universities, Inc., under cooperative agreement with the National Science Foundation. This work was supported by the University of Florida and the IBM Corp. through their Research Computing Initiative at the Northeast Regional Data Center. 